\title{
To what extent does hierarchical leadership affect health care outcomes?
}

\author{
Navindi Fernandopulle ${ }^{1^{*}}$ (1) \\ Received: 11 Apr 2020 \\ Published: 14 Sep 2021
}

\begin{abstract}
Hierarchical leadership is an antiquated practice seen commonly in health care, whereby strictly defined roles and their importance are overemphasized. This can have unintended negative consequences in a pressurised environment. In contrast, flat hierarchies are gaining popularity, as they afford the flexibility and equality that is vital in a caring environment, where no one should be afraid to raise concerns and voice their opinions.

Are hierarchical power structures inhibiting hospitals from achieving effective medical leadership and quality care? With increasing pressures on the health care system, is it time to move away from a hierarchical power structure that has been present for over 70 years? To inspire culture change, is it time to explore alternatives, such as a flat hierarchy?
\end{abstract}

Keywords: Hierarchial Leadership, Leadership In Healthcare, Patient Safety, Reverse Ward Rounds

Conflicts of Interest: None declared

Funding: None

*This work has been published under CC BY-NC-SA 1.0 license.

Copyright $($ Iran University of Medical Sciences

Cite this article as: Fernandopulle N. To what extent does hierarchical leadership affect health care outcomes?. Med J Islam Repub Iran. 2021 (14 Sep);35:117. https://doi.org/10.47176/mjiri.35.117

\section{What Is hierarchical Leadership?}

Although there has been a general consensus that effective medical leadership leads to quality care and has positive health care outcomes for patients (1), what constitutes "effective leadership" is not often clearly discussed. Currently, health care organisations, including the National Health Service (NHS), largely operate a hierarchical, pyramidal form of leadership. Hierarchical leadership can be defined as a "clearly delineated chain of command from the lowest to the highest levels within an organisation" (2). This is not uncommon in large-scale organisations, including in the corporate world. Higher up on the pyramid, the number of managers at each level decreases and their responsibilities become less concerned with day to day operations and more ab out providing an overview, "diagnosing environmental changes" and "planning responses to these changes" (3). However, in a field that is concerned with the health and wellbeing of its "customers," the patients, it can seem ironic that such decisions are taken by figures that are often away from the frontline.

Corresponding author: Dr Navindi Fernandopulle, navindif@gmail.com

1. MBBS, Bsc Gloucester Royal Hospital, Gloucester, UK

\section{How Are Flat Hierarchies Different?}

In contrast, flat hierarchies have gained increasing attention and popularity, offering a stark contrast with the traditional medical hierarchy. A flat structure is an organisational model with few or most commonly no middle management between frontline staff and decision-making executives. The number of people directly supervised by each executive is large, however, the number of people in the chain of command above is small (4). It means that the executives above the employees have more responsibility for each member of staff, as there are more people dependent on support and guidance. Flatter structures benefit from the fact that the lines of communication are shorter (5), making the firm responsive to change, unlike taller classical hierarchical structures.

\section{Leadership Styles and Hierarchy}

Hierarchy is associated in varying degrees to different styles of leadership. Steep hierarchies often demonstrate autocratic and transactional forms of leadership and are often associated with poorer health care outcomes (1).

$\uparrow$ What is "already known” in this topic:

Hierarchical leadership is prevalent in health care starting from the ward round.

$\rightarrow$ What this article adds:

Hierarchical leadership can have detrimental effects on health care outcomes and alternatives must be explored. 
Less hierarchical organisations embrace transformational leadership. In this model, the leader 'identifies the needed change, creates a vision to guide change through inspiration...' (6). Sfantou et al did a meta-analyses in 2017 to assess the association between different leadership styles and health care quality measures in nursing (1). They found that transformational leadership, which is associated with flatter hierarchies, was positively related to 'effective nursing unit organisation culture' and led to lower patient mortality rates and higher patient satisfaction. It was seen as a more collaborative approach to leadership. In contrast, transactional leadership had a weak relationship with effective nursing unit organisation culture. Therefore, it is clear that to have a positive, collaborative culture, it is important to inspire, retain, and support staff by employing a flatter hierarchy.

Intriguingly, there is some evidence in the literature to suggest an autocratic leadership might be beneficial in emergencies (1). This could perhaps be due to the leader making all the decisions without taking into account conflicting opinions of staff members, especially in a time of high stress and panic. However, it could be argued that this could feed into a 'blame culture,' fuelling division, with individuals held responsible. Furthermore, it would evoke a need to create a smooth transition out of an autocratic leadership after an emergency period has ended.

In contrast, a laissez faire leadership was negatively related with effective nursing unit organisation culture (1). It exemplifies a hands-off approach, with the leader refraining from making decisions. In a highly experienced workforce, this could be effective. However, in the health care system with frequent staff turnover and job role changes, staff would lack direction and supervision and in turn, feel more dissatisfied (7). A flat hierarchy has often been confused with a laissez faire approach by critics. A flat hierarchy simply means a structured organisation with fewer bureaucratic obstacles, where each member contributes and is valued equally. Instead of following orders, team members work alongside each other, in contrast to a complete hands-off approach.

\section{Will Flat Hierarchies Work in Health Care?}

Flat hierarchies are not new in health care. In the early 1980s, the American Nurses' Association (ANA) identified a group of hospitals that were known to be 'magnet hospitals' (8). They were referred to as such, as they had little difficulty in recruiting and retaining staff and were generally regarded as good places to work. One key feature they shared was a relatively flat nursing hierarchy with few supervisors. Aiken et al showed that such hospitals had lower mortality rates, increased patient satisfaction, lower burnout rates, and lower needle stick injuries among staff. A similar study done across the UK in 2018 revealed that patient satisfaction was directly proportional to how satisfied the staff were, and their experiences working in the NHS (9). Currently high nurse burnout and job dissatisfaction is common, with $85 \%$ registered nurses reporting not being able to complete needed nursing care because of high patient to nurse workloads. This has contributed to an increased risk of patient mortality following common surgical procedures (10). However, the magnet hospitals identified by the ANA had similar time pressures. Nevertheless, one of the major reasons why they were able to excel was because of the supportive working environment and appropriate delegation of tasks that took place. These are features that are characteristic of a flat hierarchy. For nurses to delegate tasks and ask for help, they need to feel that they can raise issues and concerns with their seniors.

A more positive organisation culture, as is found in flatter hierarchies, has consistently led to higher quality of care by empowering staff to raise patient safety concerns. Singer et al found that reduced hierarchy was associated with a 'higher level of safety climate for patients,' as it was associated with more of a group culture than a top down pyramidal hierarchy (11). This could potentially be because staff feel that they can approach seniors more easily when there is less hierarchy. This reflects that a flatter hierarchy would not only lead to a more satisfied workforce, but also have beneficial impacts on patients.

Inquiries on past failings of care, including at Bristol Royal Infirmary (BRI) and Mid Staffordshire, highlighted the role that leadership and hierarchy played. The Kennedy Inquiry into failings at BRI (12) reflected that the consultants saw themselves as having effective teams, but they saw these as their teams that they led. They were not part of the team. Also, the teams consisted of teams of like professionals, not organized around the care of the patients and were not integrated to work cross-specialty and were 'profoundly hierarchical.' The General Medical Council (GMC) guidelines sulbsequently changed to emphasize the importance of team working. Some medical schools, including St George's, University of London, began to offer interdisciplinary teaching modules, with medical students learning alongside other health care professionals to facilitate communication skills and enhance interdisciplinary team dynamic:s.

Another concern brought to light was that the hierarchical system in place at BRI made it 'difficult for the nursing staff to voice concerns and to be heard.' There was a sense of hierarchy persisting across health care professions, with nurses perceived as of lower status than doctors (13). Similar concerns were highlighted at Mid Staffordshire NHS Foundation Trust Public Inquiry. The hierarchical culture was viewed as 'bullying...oppressive' and the report drew attention to the fact that organizational culture is informed by the nature of its leadership (14). In order to preserve a positive, patient focused culture, the report advised a 'culture shift' towards one that is more approachable. The recent Gosport inquiry, initiated after approximately 600 unnecessary deaths from excess opiate usage reflected that these deaths could have been avoided had it not been for a culture where whistle blowers were largely ignored by a top-down, hierarchy (15).

It is interesting to note that despite the benefits afforded by a flatter structure, health care is one of the last remaining professions with strongly enforced hierarchies, somewhat contradictory in an organization that needs to value input from several members of a multidisciplinary team to provide patients with holistic care. One of the oldest and 
still remaining examples is the medical ward round. A senior figure, usually the consultant takes the lead. The rest of the team largely follow rather than challenge decisions. The current system, both on ward rounds and in in higher managerial roles at the boardroom expect decisions to be made not by frontline staff, but by a figure that has often spent the least amount of time with patients. On ascension of the 'hierarchical pyramid,' contact time with patients reduces. This has now begun to change, with senior figures encouraging team members to verbalize uncertainties (16). Innovative methods such as reverse ward rounds where a less experienced trainee swaps roles with the consultant who takes on an observing role have proved effective in flattening the hierarchy (16).

Intimidated juniors can be hesitant to speak up, leading to insufficient transfer of information from junior to senior members of staff, leading to serious errors and adverse events. Flattening the hierarchy is vital if we wish to convey how much we value team input. Creating an inclusive atmosphere with daily briefings and routine feedback between consultants and juniors can be another way of achieving this.

Flatter organizations provide staff with a greater degree of self-actualization (17), an attribute which is at the tip of Maslow's hierarchy of needs. Self- actualization is reaching self-fulfilment and one's maximum potential and capabilities. This is particularly important in health care, as the staff who feel more fulfilled in their roles are more optimistic (18), and this is likely to reflect in the care provided to patients.

Another major benefit of a flatter structure is that forms of communication can easily be directed to the person responsible for effecting change (19). This is in contrast to a medical hierarchy, which can act in a bureaucratic manner to 'dilute' messages. This is because of the multiple layers of middle management that needs to be crossed as well as due to the overlap in roles, and, accountability, making it difficult to know who to direct concerns and raise issues with. This runs the risk of it sometimes being too late by the time the right person has been contacted and bureaucratic delays in implementing change. For a dynamic, constantly improving health care system, we need to promote a culture where staff can raise concerns and receive an adequate response in time. By reducing the number of levels of management between executives and frontline staff, communications will improve (19). Critics of this school of thought argue to the contrary, in that the large number of staff supervised by a senior will increase in a flatter structure, hence, there is less room for concerns to be filtered and directed to the appropriate sources, and instead overburden a small number of managerial staff. Middle managers are seen as performing valuable services in streamlining issues raised and communicating strategy. However, the reduction of bureaucracy because of the smaller chain of command in a flat structure naturally mean that decisions are taken faster and change is implemented more quickly. Also, as staff take collective responsibility and are not limited by their job descriptions, there is more of an incentive to cross communicate and raise issues with members that would otherwise not have been part of one's team.

\section{What Are Some Drawbacks of a Flat Hierarchy?}

One common criticism of a flat structure is that a hierarchy is fundamental to the structure of an organization and it would be inconceivable to function without one. Critics of a flat structure argue that 'people assume hierarchy, even when there is none, and given enough time, this causes hierarchies to emerge and solidify' (20). However, it could be argued that this is more commonly an outcome of a laissez-faire or 'hands-off' approach to leadership than in a flat structure. Advocates of a flat hierarchy do not dispute the need for clearly identifiable leadership positions. Flatter structures differ from a traditional hierarchy in that they remove middle managers and increase accessibility of decision-makers to frontline staff. Polly Kettley argues that flat structures identify and reallocate 'unnecessary and costly overlaps of accountability' (19). This reduces the 'hierarchical overload' enabling decision-makers to have the right information. Help is always available, perhaps even more accessible, than if it was a hierarchy. Juniors are encouraged to approach senior most figures with their ideas and concerns, rather than having to cross several layers of middle management.

Another criticism levelled at flat structures is that it could be harder to hold individual employees accountable because of the sharing of decisions and lack of middle management. However, this could be beneficial, especially in a crisis where it is more useful to learn collectively from an incident. In the health care system, it has become increasingly common to 'victim blame' rather than hold systems accountable, as was the case with Dr Hadiza Bawa Garba. To replace a culture of fear, we need to create an organization that is transparent and rewards rather than punishes whistleblowing and problems within a system. To reach this work culture, we need a flat structure where seniors work alongside, rather than tower over juniors.

\section{Conclusion}

Hierarchical leadership can have a drastic impact on health care outcomes by affecting staff morale, which subsequently affects patient safety. It can lead to individuals being blamed, rather than encouraging a collectively responsible mentality. Effective leadership provides staff with support, with flat structures having a positive impact on the organization comparedl to a traditional hierarchy. Having multiple layers of middle management between the frontline staff and decision-making executives affecting the rate at which change can be implemented. For an open, transparent, blame-free culture within health care organizations, the staff need to be valued and seen as equals, with their concerns being taken seriously. A flatter structure enables a culture where it is easier to raise and have concerns heard, and proves to be a less intimidating arena for juniors to voice their opinions. It has worked and can work in a health care system and needs to become the norm. 
Conflict of Interests

The authors declare that they have no competing interests.

\section{References}

1. Sfantou DF, Laliotis A, Patelarou AE, Dimitra Sifaki-Pistolla MM, Patelarou E. Importance of leadership style towards quality of care measures in healthcare settings: a systematic review. Healthcare. 2017;5(4):73.

2. IGI Global. What is Hierarchical Leadership [Internet]. [cited 2019 Oct 5]. Available from: https://www.igiglobal.com/dictionary/cultivating-leaders-within/33434

3. Jago A, Vroom V. Hierarchical level and leadership style. Organizational Behavior and Human Performance. Organ Behav Hum Perform. 1977;18(1):131-45.

4. Ghiselli EE, Siegel JP. Leadership and Managerial Success in Tall and Flat Organization Structures. Pers Psychol. 1972;25(4).

5. Marshall F. Organisational structures [Internet]. The Telegraph. 2017 [cited 2019 Oct 20]. Available from: http://www.gojimo.com/organisational-structures/

6. Ross J, Kendall T. Transformational leadership [Internet]. 2016 [cited 2019 Oct 5]. Available from: https://oer.missouriwestern.edu/rsm424/chapter/transformationalleadership/

7. Frandsen B. Nursing leadership management \& leadership styles. Am Assoc Nurse Assess Coord. 2014.

8. Aiken L, Havens D, Sloane D. The Magnet nursing services recognition program: a comparison of two groups of Magnet hospitals. J Nurs Adm. 2009;39.

9. Aiken L, Sloane D, Ball J, Bruyneel L, Rafferty A, Griffiths P. Patient satisfaction with hospital care and nurses in England: an observational study. BMJ Open. 2018;8(1).

10. Ball J, Murrells T, Rafferty A, Morrow E, Griffiths P. 'Care left undone'during nursing shifts: associations with workload and perceived quality of care. BMJ Qual Saf. 2014;23(2):116-25.

11. Singer S, Lin S, Falwell A, Gaba D, Baker L. Relationship of safety climate and safety performance in hospitals. Health Serv Res. 2009;44(2p1):399-421.

12. Jones I. Bristol Royal Infirmary Inquiry [Internet]. [cited 2019 Oct 19]. Available from: https://webarchive.nationalarchives.gov.uk/20090811143810/http://w ww.bristol-inquiry.org.uk/final_report/report/index.htm

13. Francis R. Report of the Mid Staffordshire NHS Foundation Trus Public Inquiry Executive summary [Internet]. 2013. Available from: https://assets.publishing.service.gov.uk/government/uploads/system/u ploads/attachment_data/file/279124/0947.pdf

14. Learning from Gosport [Internet]. Department of Health and Social Care. 2018 [cited 2019 Oct 19]. Available from: https://assets.publishing.service.gov.uk/government/uploads/system/u ploads/attachment data/file/758062/government-response-to-gosportindependent-panel-report.pdf

15. Improving teams in healthcare [Internet]. Royal College of Emergency Medicine. 2017 [cited 2019 Oct 5]. Available from: https:/www.rcem.ac.uk/docs/External Guidance/ITIH R2 Final.pdf

16. Leadership and management standards for medical professionals [Internet]. Faculty of Leadership and Management. 2016 [cited 2019 Oct 5]. Available from: https://www.fmlm.ac.uk/individual-standards

17. West M, Armit K, Loewenthal L. Leadership and leadership development in healthcare: The evidence base. The Kings Fund. 2015.

18. Maslow AH. Motivation and personality. 3rd ed. Delhi, India: Pearson Education; 1987.

19. Kettley P. Is flatter better? Delayering the management hierarchy [Internet]. Institute for Employment Studies. 1995 [cited 2019 Oct 19]. Available from: https://www.employmentstudies.co.uk/system/files/resources/files/290.pdf

20. Why companies struggle to build flat hierarchies. Available from: https://pressreleases.responsesource.com/news/96485/whycompanies-struggle-to-build-flat-hierarchies/ 